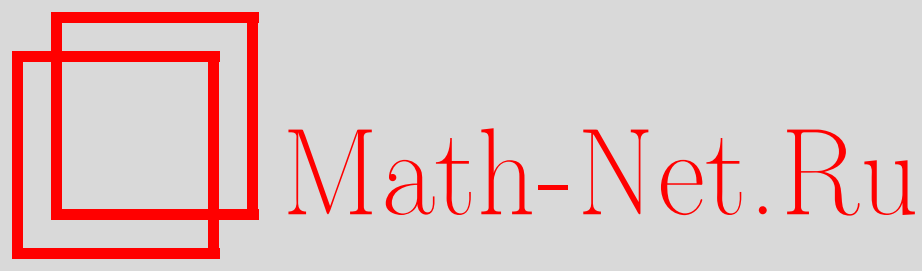

У. Джайрук, С. Ю-Конг, М. Танаситтикосол, О лагранжевой структуре модели золотой рыбки Калоджеро, TMФ, 2015, том 183, номер 2, 254-273

DOI: https://doi.org/10.4213/tmf8800

Использование Общероссийского математического портала Math-Net.Ru подразумевает, что вы прочитали и согласны с пользовательским соглашением http://www.mathnet.ru/rus/agreement

Параметры загрузки :

IP : 54.174 .149 .18

26 апреля 2023 г., 13:56:43

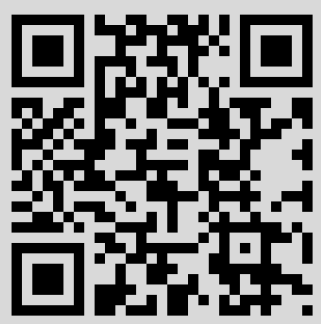


Том 183, № 2

май, 2015

(C) 2015 г. У. Джайрук*, С. Ю-Конг*† , М. Танаситтикосол*

\title{
О ЛАГРАНЖЕВОЙ СТРУКТУРЕ МОДЕЛИ ЗОЛОТОЙ РЫБКИ КАЛОДЖЕРО
}

\begin{abstract}
Из анзаца пары Лакса получена рациональная система золотой рыбки Калоджеро с дискретным временем. Лагранжианы с дискретным временем для этой системы имеют структуру 1-формы с дискретным временем подобно лагранжианам систем Калоджеро-Мозера и Руджинарса-Шнайдера с дискретным временем. Иерархия лагранжианов для этой системы получается в результате двухшагового перехода к непрерывному пределу. Как и ожидалось, лагранжиан с непрерывным временем сохраняет структуру 1-формы. Установлена связь с решеточными системами Кадомцева-Петвиашвили.
\end{abstract}

Ключевые слова: золотая рыбка Калоджеро, многовременные лагранжевы 1-формы, условия замыкания.

DOI: $10.4213 / \operatorname{tmf} 8800$

\section{1. ВВЕДЕНИЕ}

Свойство многомерной совместности играет очень важную роль в понятии интегрируемости дискретных систем. Говоря коротко, обнаружено следующее свойство произвольной $D$-мерной дискретной системы: систему более высокой размерности (в пространстве и времени) можно совместным образом построить из подсистемы более низкой размерности (в пространстве и времени). Число измерений $D$ можно устремить к бесконечности, в этом случае получим бесконечный набор совместных подсистем.

Согласно принципу наименьшего действия в классической механике действие системы стационарно на классической траектории в пространстве, состоящем из независимой переменной (независимых переменных) и зависимой переменной (зависимых переменных). В таком случае можно задать вопрос об аналоге принципа наименьшего действия для систем, обладающих многомерной совместностью. Мы рассматриваем не только траекторию в подпространстве, состоящем из зависимой

${ }^{*}$ Theoretical and Computational Physics Group, Department of Physics, Faculty of Science, King Mongkut's University of Technology Thonburi, Thailand. E-mail: ajairuk@gmail.com

†Theoretical and Computational Science Center, Faculty of Science, King Mongkut's University of Technology Thonburi, Thailand. E-mail: syookong@gmail.com

${ }^{\ddagger}$ Ratchaburi Campus, King Mongkut’s University of Technology Thonburi, Thailand 
переменной (зависимых переменых) и независимой переменной (независимых переменных), но также и подпространство независимой переменной (независимых переменных). Недавно Лоббом и Нийхофом [1]-[3] была предложена теория, названная теорией многообразия лагранжианов, в которой делается попытка явно ответить на этот вопрос для случаев $D=2$ [1], [2] и $D=3$ [3]. Ключевым в этой теории является тот факт, что действие системы инвариантно относительно вариации независимых переменных, это приводит к интересному соотношению, названному соотношением замыкания. Данное соотношение можно рассматривать в качестве вариации многомерной совместности в лагранжевой формулировке. Для случая $D=1$ была изучена конкретная модель как с дискретным, так и с непрерывным временем [4], названная рациональной системой Калоджеро-Мозера, которая представляет собой систему многих тел в одномерии с дальнодействием [5], [6]. В этом случае лагранжианы имеют структуру 1-формы. Вскоре после этого была подробно изучена лагранжева структура рациональной системы Рейсенарса-Шнайдера (которая считается релятивистской версией системы Калоджеро-Мозера) [7]. В работе [8] развита общая математическая теория, основанная на ключевых моментах, изложенных в работах [4], [7]. Случай одномерной системы многих тел с взаимодействием ближайших соседей, названной системой типа Тоды, также изучался на дискретном уровне [9].

В настоящей статье рассматривается система, названная рациональной системой золотой рыбки Калоджеро [10], что завершает обширное описание теории лагранжианов со структурой 1-формы для интегрируемых систем многих тел с дальнодействием. Интересно отметить, что систему золотой рыбки Калоджеро можно получить из системы Рейсенарса-Шнайдера, устремив релятивистские параметры к бесконечности (при приближении релятивистских параметров к нулю система переходит в систему Калоджеро-Мозера). Статья организована следующим образом. В разделе 2 подробно описывается система с дискретным временем. Считается вариация действия с дискретным временем, состоящего из дискретных кривых, что приводит к уравнению Эйлера-Лагранжа с дискретным временем, а также к соотношению замыкания. В разделе 3 найден первый непрерывный предел, названный асимметричным, переводящий систему на полудискретный уровень. В разделе 4 осуществлен второй непрерывный предел, устраняющий оставшуюся дискретную переменную и переводящий систему на полностью непрерывный уровень. В разделе 5 с использованием структуры точного решения системы устанавливается связь с решеточной системой Кадомцева-Петвиашвили. Результаты статьи обсуждаются в разделе 6.

\section{2. СИСТЕМА ЗОЛОТОЙ РЫБКИ С ДИСКРЕТНЫМ ВРЕМЕНЕМ И КОММУТИРУЮЩИЕ ПОТОКИ}

Построим систему золотой рыбки Калоджеро с дискретным временем. Сначала рассмотрим следующую систему линейных уравнений:

$$
\begin{aligned}
\mathbf{L}_{\kappa} \phi & =\zeta \phi \\
\mathbf{M}_{\kappa} \phi & =\widetilde{\phi} \\
\mathbf{N}_{\kappa} \phi & =\widehat{\phi},
\end{aligned}
$$

где $\phi=\phi(n, m)$ - вектор-функция, $\zeta$ - собственное значение. Здесь переменные $(n, m)$ являются дискретными временами, при этом $\widetilde{\phi}=\phi(n+1, m)$ и $\widehat{\phi}=\phi(n, m+1)$. 
В рациональном случае матрицы $\mathbf{L}_{\kappa}, \mathbf{M}_{\kappa}$ и $\mathbf{N}_{\kappa}$ можно взять в следующем виде:

$$
\begin{aligned}
\mathbf{L}_{\kappa} & =\frac{h h^{\mathrm{T}}}{\kappa}+\mathbf{L}_{0}, \\
\mathbf{M}_{\kappa} & =\frac{\tilde{h} h^{\mathrm{T}}}{\kappa}+\mathbf{M}_{0}, \\
\mathbf{N}_{\kappa} & =\frac{\hat{h} h^{\mathrm{T}}}{\kappa}+\mathbf{N}_{0}
\end{aligned}
$$

и

$$
\begin{aligned}
\mathbf{L}_{0} & =\sum_{i, j=1}^{N} h_{i} h_{j} E_{i j}, \\
\mathbf{M}_{0} & =\sum_{i, j=1}^{N} \frac{\tilde{h}_{i} h_{j}}{\tilde{x}_{i}-x_{j}} E_{i j}, \\
\mathbf{N}_{0} & =\sum_{i, j=1}^{N} \frac{\hat{h}_{i} h_{j}}{\hat{x}_{i}-x_{j}} E_{i j},
\end{aligned}
$$

где $x_{i}$ - положение $i$-й частицы, $N$ - число частиц в системе, $E_{i j}$ - матрица с элементами $\left(E_{i j}\right)_{k l}=\delta_{i k} \delta_{j l}$, переменные $h_{i}=h_{i}(n, m)$ вспомогательные, а также введены обозначения $x_{i}=x_{i}(n, m)$ :

$$
\begin{aligned}
& x_{i}(n+1, m)=\tilde{x}_{i}(\text { сдвиг вперед в tilde-направлении }), \\
& x_{i}(n-1, m)={\underset{\sim}{i}}_{i}(\text { сдвиг назад в tilde-направлении }), \\
& x_{i}(n, m+1)=\hat{x}_{i}(\text { сдвиг вперед в hat-направлении }), \\
& x_{i}(n, m-1)=x_{i}(\text { сдвиг назад в hat-направлении }),
\end{aligned}
$$

при этом переменная $\kappa$ является дополнительным спектральным параметром.

Рассмотрим совместность системы уравнений (2.1).

2.1. Первый дискретный поток. Совместность уравнений (2.1а) и (2.1б) приводит к соотношению

$$
\begin{aligned}
\widetilde{\mathbf{L}}_{\kappa} \mathbf{M}_{\kappa} & =\mathbf{M}_{\kappa} \mathbf{L}_{\kappa} \\
\left(\frac{\tilde{h} \tilde{h}^{\mathrm{T}}}{\kappa}+\widetilde{\mathbf{L}}_{0}\right)\left(\frac{\tilde{h} h^{\mathrm{T}}}{\kappa}+\mathbf{M}_{0}\right) & =\left(\frac{\tilde{h} h^{\mathrm{T}}}{\kappa}+\mathbf{M}_{0}\right)\left(\frac{h h^{\mathrm{T}}}{\kappa}+\mathbf{L}_{0}\right) .
\end{aligned}
$$

Рассматривая коэффициент перед $1 / \kappa^{2}$, получим соотношение

$$
\sum_{j=1}^{N} \tilde{h}_{j}^{2}=\sum_{j=1}^{N} h_{j}^{2},
$$

а для определения коэффициента перед $1 / \kappa$ получаем собой следующее уравнение:

$$
\widetilde{\mathbf{L}}_{0} \tilde{h} h^{\mathrm{T}}+\tilde{h} \tilde{h}^{\mathrm{T}} \mathbf{M}_{0}=\mathbf{M}_{0} h h^{\mathrm{T}}+\tilde{h} h^{\mathrm{T}} \mathbf{L}_{0} .
$$

Для оставшейся части соотношения $(2.2 \mathrm{a})$ имеем

$$
\widetilde{\mathbf{L}}_{0} \mathbf{M}_{0}=\mathbf{M}_{0} \mathbf{L}_{0} .
$$


Уравнения (2.2в) и (2.2г) порождают одинаковое семейство уравнений

$$
\sum_{j=1}^{N} \frac{\tilde{h}_{j}^{2}}{\tilde{x}_{j}-x_{l}}=\sum_{j=1}^{N} \frac{h_{j}^{2}}{\tilde{x}_{i}-x_{j}}
$$

для всех $i, j=1,2, \ldots, N$. Поскольку две стороны уравнения (2.2д) зависят от различных внешних индексов, можно написать систему спаренных уравнений:

$$
\begin{aligned}
& \sum_{j=1}^{N} \frac{\tilde{h}_{j}^{2}}{\tilde{x}_{j}-x_{l}}=-p \quad \forall l, \\
& \sum_{j=1}^{N} \frac{h_{j}^{2}}{\tilde{x}_{i}-x_{j}}=-p \quad \forall i
\end{aligned}
$$

где $p=p(n)$ не зависит от индексов частиц, но все же может быть функцией дискретного времени $n$.

Чтобы определить функцию $h_{i}$, воспользуемся интерполяционной формулой Лагранжа. Рассмотрим $2 N$ не совпадающих комплексных чисел $x_{k}$ и $y_{k}$, где $k=$ $1,2, \ldots, N$. Тогда справедлива следующая формула:

$$
\prod_{k=1}^{N} \frac{\xi-x_{k}}{\xi-y_{k}}=1+\sum_{k=1}^{N} \frac{1}{\xi-y_{k}} \frac{\prod_{j=1}^{N}\left(y_{k}-x_{j}\right)}{\prod_{j=1, j \neq k}^{N}\left(y_{k}-y_{j}\right)}
$$

Как следствие выпишем систему уравнений

$$
-1=\sum_{k=1}^{N} \frac{1}{x_{i}-y_{k}} \frac{\prod_{j=1}^{N}\left(y_{k}-x_{j}\right)}{\prod_{j=1, j \neq k}^{N}\left(y_{k}-y_{j}\right)}, \quad i=1,2, \ldots, N
$$

которая получается подстановкой $\xi=x_{i}$ в уравнение $(2.23)$.

Используя систему (2.2и), получим

$$
\begin{aligned}
& h_{j}^{2}=p \frac{\prod_{i=1}^{N}\left(x_{j}-\tilde{x}_{i}\right)}{\prod_{j \neq i}^{N}\left(x_{j}-x_{i}\right)}, \\
& \tilde{h}_{j}^{2}=-p \frac{\prod_{i=1}^{N}\left(\tilde{x}_{j}-x_{i}\right)}{\prod_{j \neq i}^{N}\left(\tilde{x}_{j}-\tilde{x}_{i}\right)}
\end{aligned}
$$

для $j=1,2, \ldots, N$. Приравняв уравнения $(2.2 \mathrm{\kappa})$ и $(2.2 л)$, приходим к следующей системе уравнений:

$$
-\frac{p}{p} \frac{x_{i}-\tilde{x}_{i}}{x_{i}-{\underset{\sim}{x}}_{i}}=\prod_{\substack{j=1 \\ j \neq i}}^{N} \frac{x_{i}-\underline{x}_{j}}{x_{i}-\tilde{x}_{j}}
$$

Пусть для простоты $p$ - постоянная величина, тогда (2.2м) является обычным уравнением движения золотой рыбки Калоджеро с дискретным временем в tilde-направлении (см. [11]). 
2.2. Второй дискретный поток. Рассмотрим совместность уравнений (2.1a) и $(2.16)$ :

$$
\begin{aligned}
\widehat{\mathbf{L}}_{\kappa} \mathbf{N}_{\kappa} & =\mathbf{N}_{\kappa} \mathbf{L}_{\kappa} \\
\left(\frac{\hat{h} \hat{h}^{\mathrm{T}}}{\kappa}+\widehat{\mathbf{L}}_{0}\right)\left(\frac{\hat{h} h^{\mathrm{T}}}{\kappa}+\mathbf{N}_{0}\right) & =\left(\frac{\hat{h} h^{\mathrm{T}}}{\kappa}+\mathbf{N}_{0}\right)\left(\frac{h h^{\mathrm{T}}}{\kappa}+\mathbf{L}_{0}\right) .
\end{aligned}
$$

При этом получим соотношения

$$
\sum_{j=1}^{N} \hat{h}_{j}^{2}=\sum_{j=1}^{N} h_{j}^{2}
$$

и семейство уравнений

$$
\sum_{j=1}^{N} \frac{\hat{h}_{j}^{2}}{\hat{x}_{j}-x_{l}}=\sum_{j=1}^{N} \frac{h_{j}^{2}}{\hat{x}_{i}-x_{j}}
$$

для всех $i, j=1,2, \ldots, N$. Воспользовавшись теми же соображениями, что и в предыдущем случае, получим следующую систему уравнений:

$$
\begin{array}{ll}
\sum_{j=1}^{N} \frac{\hat{h}_{j}^{2}}{\hat{x}_{j}-x_{l}}=-q & \forall l, \\
\sum_{j=1}^{N} \frac{h_{j}^{2}}{\hat{x}_{i}-x_{j}}=-q & \forall i,
\end{array}
$$

но параметр $q=q(m)$ при этом будет другим. Применив интерполяционную формулу Лагранжа, получим

$$
\begin{aligned}
& h_{j}^{2}=q \frac{\prod_{i=1}^{N}\left(x_{j}-\hat{x}_{i}\right)}{\prod_{j \neq i}^{N}\left(x_{j}-x_{i}\right)}, \\
& \hat{h}_{j}^{2}=-q \frac{\prod_{i=1}^{N}\left(\hat{x}_{j}-x_{i}\right)}{\prod_{j \neq i}^{N}\left(\hat{x}_{j}-\hat{x}_{i}\right)}
\end{aligned}
$$

для $i=1,2, \ldots, N$, а также семейство уравнений

$$
-\frac{q}{q} \frac{x_{i}-x_{i}}{x_{i}-\hat{x}_{i}}=\prod_{\substack{j=1 \\ j \neq i}}^{N} \frac{x_{i}-\hat{x}_{j}}{x_{i}-x_{j}} .
$$

Пусть $q$ также является постоянной, тогда уравнение (2.33) является уравнением движения системы золотой рыбки Калоджеро с дискретным временем в hat-направлении.

2.3. Коммутативность потоков. Условие совместимости уравнений и $(2.1$ в) имеет вид

$$
\begin{aligned}
\widehat{\mathbf{M}}_{\kappa} \mathbf{N}_{\kappa} & =\widetilde{\mathbf{N}}_{\kappa} \mathbf{M}_{\kappa} \\
\left(\frac{\hat{\tilde{h}} \hat{h}^{\mathrm{T}}}{\kappa}+\widehat{\mathbf{M}}_{0}\right)\left(\frac{\hat{h} h^{\mathrm{T}}}{\kappa}+\mathbf{N}_{0}\right) & =\left(\frac{\tilde{\tilde{h}} \tilde{h}^{\mathrm{T}}}{\kappa}+\widetilde{\mathbf{N}}_{0}\right)\left(\frac{\tilde{h} h^{\mathrm{T}}}{\kappa}+\mathbf{M}_{0}\right) .
\end{aligned}
$$


Уравнение (2.4a) порождает соотношение

$$
\sum_{j=1}^{N} \tilde{h}_{j}^{2}=\sum_{j=1}^{N} \hat{h}_{j}^{2}
$$

которое можно считать следствием первых двух соотношений, полученных для переменной $h_{i}$. Кроме того, имеем

$$
\begin{aligned}
\hat{\tilde{h}} \hat{h}^{\mathrm{T}} \mathbf{N}_{0}-\hat{\tilde{h}} \tilde{h}^{\mathrm{T}} \mathbf{M}_{0} & =\widetilde{\mathbf{N}}_{0} \tilde{h} h^{\mathrm{T}}-\widehat{\mathbf{M}}_{0} \hat{h} h^{\mathrm{T}} \\
\widehat{\mathbf{M}}_{0} \mathbf{N}_{0} & =\widetilde{\mathbf{N}}_{0} \mathbf{M}_{0}
\end{aligned}
$$

что порождает семейство уравнений

$$
\sum_{j=1}^{N}\left(\frac{\hat{h}_{j}^{2}}{\hat{\tilde{x}}_{i}-\hat{x}_{j}}-\frac{\tilde{h}_{j}^{2}}{\hat{\tilde{x}}_{i}-\tilde{x}_{j}}\right)=\sum_{j=1}^{N}\left(\frac{\tilde{h}_{j}^{2}}{\tilde{x}_{j}-x_{l}}-\frac{\hat{h}_{j}^{2}}{\hat{x}_{j}-x_{l}}\right) .
$$

И снова это семейство есть не что иное, как следствие уравнений $(2.2 \mathrm{\kappa}),(2.2 \pi),(2.3 \mathrm{e})$ и $(2.3$ ж).

Приравнивая уравнения $(2.2 \mathrm{k})$ и $(2.3 \mathrm{e})$, а также уравнения $(2.2 л)$ и $(2.3 ж)$, получим

$$
\begin{aligned}
& \frac{p}{q}=\prod_{j=1}^{N} \frac{x_{i}-\hat{x}_{j}}{x_{i}-\tilde{x}_{j}} \\
& \frac{p}{q}=\prod_{j=1}^{N} \frac{x_{i}-x_{j}}{x_{i}-{\underset{\sim}{j}}_{j}} .
\end{aligned}
$$

Используя уравнения движения (2.2м) и (2.33), выведем два других соотношения:

$$
\begin{aligned}
-\frac{p}{q} & =\prod_{j=1}^{N} \frac{x_{i}-\hat{x}_{j}}{x_{i}-{\underset{\sim}{\sim}}_{j}}, \\
-\frac{p}{q} & =\prod_{j=1}^{N} \frac{x_{i}-x_{j}}{x_{i}-\tilde{x}_{j}} .
\end{aligned}
$$

Эти уравнения можно считать ограничениями, описывающими связь двух дискретных потоков в центре решетки (см. рис. 1). Приравнивая уравнения (2.4е) и $(2.4 ж)$, а также уравнения $(2.43)$ и $(2.4$ и), приходим к уравнениям движения золотой рыбки Калоджеро с дискретными временами

$$
\prod_{j=1}^{N} \frac{x_{i}-\tilde{x}_{j}}{x_{i}-\underline{x}_{j}}=\prod_{j=1}^{N} \frac{x_{i}-\hat{x}_{j}}{x_{i}-\underline{x}_{j}}
$$

которые выражают совместность с семейством обыкновенных разностных уравнений. 


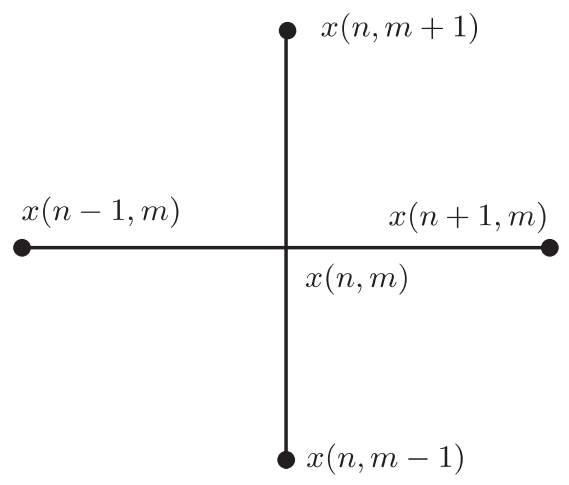

Рис. 1. Структура решетки состоит из двух дискретных потоков: $(n, m)$. Горизонтальная и вертикальная линии описываются уравнениями движения в tilde- и hat-направлениях соответственно. Ограничения описывают связь двух дискретных потоков в четырех углах вокруг центра.

2.4. Точное решение. Сначала рассмотрим решение для tilde-направления. Матрицы $\mathbf{M}_{0}$ и $\mathbf{L}_{0}$ перепишем в виде

$$
\begin{aligned}
\widetilde{\mathbf{X}} \mathbf{M}_{0}-\mathbf{M}_{0} \mathbf{X} & =\tilde{h} h^{\mathrm{T}}, \\
\mathbf{L}_{0} & =h h^{\mathrm{T}},
\end{aligned}
$$

где $\mathbf{X}=\sum_{i=1}^{N} x_{i} E_{i i}$ - диагональная матрица. Из уравнений Лакса (2.2в) и (2.2г) получим соотношения

$$
\begin{aligned}
\widetilde{\mathbf{L}}_{0} \mathbf{M}_{0} & =\mathbf{M}_{0} \mathbf{L}_{0}, \\
\widetilde{\mathbf{L}}_{0} \tilde{h}-\mathbf{M}_{0} h & =-p \tilde{h}, \\
h^{\mathrm{T}} \mathbf{L}_{0}-\tilde{h}^{\mathrm{T}} \mathbf{M}_{0} & =-p h^{\mathrm{T}} .
\end{aligned}
$$

Теперь факторизуем матрицы Лакса следующим образом:

$$
\mathbf{L}_{0}=\mathbf{U} \mathbf{\Lambda} \mathbf{U}^{-1}, \quad \mathbf{M}_{0}=\widetilde{\mathbf{U}} \mathbf{U}^{-1},
$$

где $\mathbf{U}$ - обратимая $(N \times N)$-матрица, а матрица $\boldsymbol{\Lambda}$ является постоянной и $\tilde{\boldsymbol{\Lambda}}=\boldsymbol{\Lambda}$. Очевидно, если матрица $\mathbf{L}_{0}$ диагонализуема, то $\boldsymbol{\Lambda}$ - просто диагональная матрица, на диагонали которой стоят собственные значения матрицы $\mathbf{L}_{0}$. Далее, введем обозначения

$$
\mathbf{Y}=\mathbf{U}^{-1} \mathbf{X U}, \quad \mathbf{r}=\mathbf{U}^{-1} \cdot h, \quad \mathbf{s}^{\mathrm{T}}=h^{\mathrm{T}} \cdot \mathbf{U}
$$

и получим из уравнений $(2.7)$ и $(2.8)$ соотношения

$$
(p I+\boldsymbol{\Lambda}) \cdot \tilde{\mathbf{r}}=\mathbf{r}, \quad \mathbf{s}^{\mathrm{T}} \cdot(p I+\boldsymbol{\Lambda})=\tilde{\mathbf{s}}^{\mathrm{T}},
$$

где $I$ - единичная матрица. Из уравнения (2.6а) имеем

$$
\tilde{\mathbf{Y}}-\mathbf{Y}=\tilde{\mathbf{r}} \mathbf{s}^{\mathrm{T}} .
$$

Диаду $\mathbf{r s}^{\mathrm{T}}$ можно исключить из уравнения (2.11) с помощью выражений (2.10), получив в результате уравнение

$$
\widetilde{\mathbf{Y}}=\mathbf{Y}+\frac{\boldsymbol{\Lambda}}{p I+\boldsymbol{\Lambda}} .
$$


После $n$ дискретных шагов найдем

$$
\mathbf{Y}(n, m)=\mathbf{Y}(0, m)+\frac{n \boldsymbol{\Lambda}}{p I+\boldsymbol{\Lambda}} .
$$

Автоматически получим, что решение в hat-направлении имеет вид

$$
\mathbf{Y}(n, m)=\mathbf{Y}(n, 0)+\frac{m \boldsymbol{\Lambda}}{q I+\boldsymbol{\Lambda}} .
$$

Комбинируя уравнения (2.13) и (2.14), получим полное решение системы

$$
\mathbf{Y}(n, m)=\mathbf{Y}(0,0)+\frac{n \boldsymbol{\Lambda}}{p I+\boldsymbol{\Lambda}}+\frac{m \boldsymbol{\Lambda}}{q I+\boldsymbol{\Lambda}},
$$

при этом $x_{i}(n, m)$ можно определить с помощью собственных значений матрицы $\mathbf{Y}(n, m)$.

2.5. Дискретные действия. Мы пришли к выводу, что уравнения движения в tilde-направлении (горизонтальная дискретная кривая на рис. 1) появляются вследствие вариации дискретного действия

$$
S_{H}=\mathscr{L}_{(p)}(\mathbf{x}, \tilde{\mathbf{x}})+\mathscr{L}_{(p)}(\mathbf{x}, \underset{\sim}{\mathbf{x}})
$$

что приводит к уравнению

$$
\delta S_{H}=0 \Rightarrow \frac{\partial \mathscr{L}_{(p)}}{\partial \tilde{x}_{i}}+\frac{\widetilde{\partial \mathscr{L}_{(p)}}}{\partial x_{i}}=0
$$

где

$$
\mathscr{L}_{(p)}=\sum_{i, j=1}^{N}\left(x_{i}-\tilde{x}_{j}\right) \ln \left(x_{i}-\tilde{x}_{j}\right)+\ln |p| \sum_{i=1}^{N}\left(x_{i}-\tilde{x}_{i}\right) .
$$

Уравнение (2.16б) порождает уравнения движения с дискретным временем в tilde-направлении (2.2м). Существуют уравнения движения и в hat-направлении (вертикальная дискретная кривая на рис. 1), которые появляются вследствие вариации дискретного действия

$$
S_{V}=\mathscr{L}_{(q)}(\mathbf{x}, \hat{\mathbf{x}})+\mathscr{L}_{(q)}(\mathbf{x}, \mathbf{x})
$$

что приводит к уравнению

$$
\delta S_{V}=0 \Rightarrow \frac{\partial \mathscr{L}_{(q)}}{\partial \hat{x}_{i}}+\frac{\widehat{\partial \mathscr{L}_{(q)}}}{\partial x_{i}}=0
$$

где

$$
\mathscr{L}_{(q)}=\sum_{i, j=1}^{N}\left(x_{i}-\hat{x}_{j}\right) \ln \left(x_{i}-\hat{x}_{j}\right)+\ln |q| \sum_{i=1}^{N}\left(x_{i}-\hat{x}_{i}\right) .
$$

Уравнение (2.16г) порождает уравнения движения с дискретным временем в tildeнаправлении (2.33). Кроме того, есть еще четыре различных дискретных действия, 


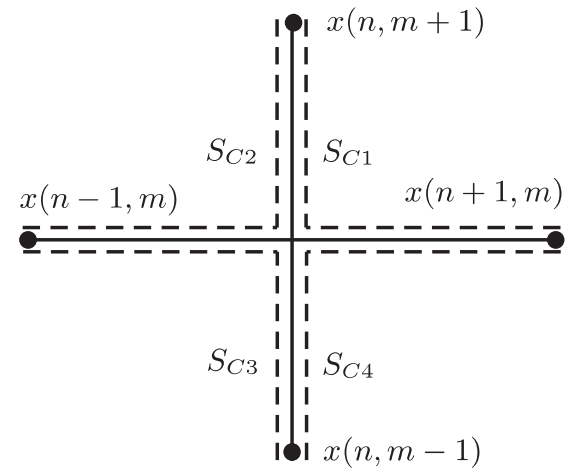

a

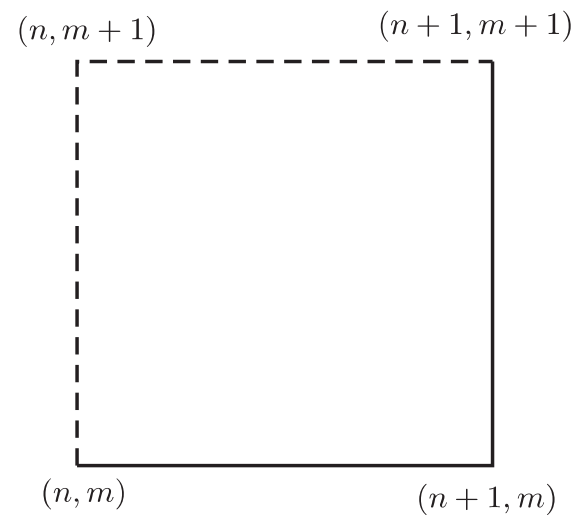

б

Рис. 2. Дискретные действия вокруг центра решетки (а). Деформация дискретных кривых в пространстве независимых переменных (б).

соответствующих двум различным дискретным кривым, соединяющимся в центре, как показано на рис. 2а:

$$
\begin{aligned}
& S_{C 1}=\mathscr{L}_{(p)}(\tilde{\mathbf{x}}, \mathbf{x})+\mathscr{L}_{(q)}(\mathbf{x}, \hat{\mathbf{x}}), \\
& S_{C 2}=\mathscr{L}_{(p)}(\underset{\sim}{\mathbf{x}}, \mathbf{x})+\mathscr{L}_{(q)}(\mathbf{x}, \hat{\mathbf{x}}), \\
& S_{C 3}=\mathscr{L}_{(p)}(\underset{\sim}{\mathbf{x}}, \mathbf{x})+\mathscr{L}_{(q)}\left(\mathbf{x}, \mathbf{x}_{\sim}\right), \\
& S_{C 4}=\mathscr{L}_{(p)}(\tilde{\mathbf{x}}, \mathbf{x})+\mathscr{L}_{(q)}\left(\mathbf{x}, \mathbf{x}_{\sim}\right) .
\end{aligned}
$$

Вариация этих четырех действий дает не что иное, как ограничивающие уравнения.

Еще одной важной характеристикой этих дискретных лагранжианов является соотношение замыкания

$$
\widehat{\mathscr{L}_{(p)}(\mathbf{x}, \tilde{\mathbf{x}})}-\mathscr{L}_{(p)}(\mathbf{x}, \tilde{\mathbf{x}})-\widehat{\mathscr{L}_{(q)}(\mathbf{x}, \hat{\mathbf{x}})}+\mathscr{L}_{(q)}(\mathbf{x}, \hat{\mathbf{x}})=0
$$

которое является непосредственным результатом вариации дискретной кривой в пространстве независимых переменных $(n, m)$ (см. также [4], [7]). Справедливость уравнения (2.16л) можно продемонстрировать с помощью уравнений движения $(2.2 \mathrm{M})$ и (2.33). Соотношение замыкания гарантирует инвариантность действия относительно локальной деформации дискретной кривой (см. рис. 2б).

ЗАмЕчАниЕ 1. Из лагранжианов (2.16в) и (2.16е) можно определить переменные-импульсы

$$
\begin{gathered}
\mathrm{p}_{i}=-\frac{\partial \mathscr{L}_{(p)}}{\partial \tilde{x}_{i}}=\sum_{j=1}^{N} \ln \left(x_{j}-\tilde{x}_{i}\right)+1+\ln |p|, \\
\pi_{i}=-\frac{\partial \mathscr{L}_{(q)}}{\partial \hat{x}_{i}}=\sum_{j=1}^{N} \ln \left(x_{j}-\hat{x}_{i}\right)+1+\ln |q|,
\end{gathered}
$$


связанные соответственно с tilde- и hat-направлениями. Используя эти соотношения, можно переписать уравнение $(2.2 \mathrm{~K})$ в виде

$$
h_{k}^{2}=\frac{e^{\mathrm{p}_{k}-1}}{\prod_{j \neq k}\left(x_{k}-x_{j}\right)},
$$

а уравнение (2.3е) в виде

$$
h_{k}^{2}=\frac{e^{\pi_{k}-1}}{\prod_{j \neq k}\left(x_{k}-x_{j}\right)} .
$$

Гамильтониан системы представляется выражением

$$
\mathscr{H}=\operatorname{Tr} \mathbf{L}_{0}=\sum_{k=1}^{N} h_{k}^{2} .
$$

Тогда уравнения (2.2б), (2.3б) и (2.4б) принимают вид

$$
\begin{array}{llll}
\sum_{k=1}^{N} h_{k}^{2}=\sum_{k=1}^{N} \tilde{h}_{k}^{2} & \mapsto & \mathscr{H}=\widetilde{\mathscr{H}}, \\
\sum_{k=1}^{N} h_{k}^{2}=\sum_{k=1}^{N} \hat{h}_{k}^{2} & \mapsto & \mathscr{H}=\widehat{\mathscr{H}}, \\
\sum_{k=1}^{N} \tilde{h}_{k}^{2}=\sum_{k=1}^{N} \hat{h}_{k}^{2} & \mapsto & \widetilde{\mathscr{H}}=\widehat{\mathscr{H}} .
\end{array}
$$

Уравнения (2.22) и (2.23) представляют собой закон сохранения энергии в tildeи hat-направлениях, поскольку оба дискретных потока используют одну и ту же матрицу $\mathbf{L}_{0}$. Уравнение (2.24) можно считать дискретным аналогом условия коммутирования потоков.

\section{3. ЧАСТИЧНО-НЕПРЕРЫВНЫЙ ПРЕДЕЛ}

Рассмотрим непрерывный предел системы золотой рыбки Калоджеро с дискретным временем, которая исследовалась в разделе 2. Поскольку существуют два дискретных времени $(n, m)$, можно непосредственно осуществить непрерывный предел по каждой из этих переменных и получить в результате обычную систему золотой рыбки Калоджеро с непрерывным временем [11]. Перейдем теперь к непрерывному пределу другого типа, а именно к асимметричному пределу. Чтобы приступить к нахождению этого предела, введем новое дискретное время $\mathrm{N}=n+m$ и с помощью этой новой переменной зададим семейство следующих преобразований переменных:

$$
\begin{aligned}
x(n, m) & \mapsto \mathrm{x}(\mathrm{N}, m)=: \mathrm{x}, \\
\tilde{x}=x(n+1, m) & \mapsto \mathrm{x}(\mathrm{N}+1, m)=: \overline{\mathrm{x}}, \\
\hat{x}=x(n, m+1) & \mapsto \mathrm{x}(\mathrm{N}+1, m+1)=: \hat{\overline{\mathrm{x}}}, \\
\hat{\tilde{x}}=x(n+1, m+1) & \mapsto \mathrm{x}(\mathrm{N}+2, m+1)=: \hat{\overline{\overline{\mathrm{x}}}}
\end{aligned}
$$

Введем также $\varepsilon=p-q$ и $\varepsilon m=\tau$, после чего устремим $n \rightarrow-\infty, m \rightarrow \infty, \varepsilon \rightarrow 0$, считая $\mathrm{N}$ и $\tau$ фиксированными. 
Сначала рассмотрим асимметричный предел в точном решении, представленном выражением (2.15). Перепишем это решение в новых переменных:

$$
\begin{aligned}
\mathbf{Y}(n, m) & \mapsto \mathbf{Y}(\mathrm{N}, m)=\mathbf{Y}(0,0)+\frac{\mathrm{N} \boldsymbol{\Lambda}}{p I+\boldsymbol{\Lambda}}+\frac{m \varepsilon \boldsymbol{\Lambda}}{(p+\boldsymbol{\Lambda})^{2}(1-\varepsilon /(p+\boldsymbol{\Lambda}))}, \\
\lim _{\substack{m \rightarrow \infty \\
\varepsilon \rightarrow 0 \\
\varepsilon m \rightarrow \tau}} \mathbf{Y}(\mathrm{N}, m) & \mapsto \mathbf{Y}(\mathrm{N}, \tau)=\mathbf{Y}(0,0)+\frac{\mathrm{N} \boldsymbol{\Lambda}}{p I+\boldsymbol{\Lambda}}+\frac{\tau \boldsymbol{\Lambda}}{(p I+\boldsymbol{\Lambda})^{2}} .
\end{aligned}
$$

Сдвиг положения частицы в hat-направлении описывается формулами

$$
\begin{aligned}
& \hat{\overline{\mathrm{x}}}=\mathrm{x}(\mathrm{N}+1, m+1) \mapsto \hat{\overline{\mathrm{x}}}=\mathrm{x}(\mathrm{N}+1, \tau+\varepsilon), \\
& \underline{\mathrm{x}}=\mathrm{x}(\mathrm{N}-1, m-1) \mapsto \underline{\mathrm{x}}=\mathrm{x}(\mathrm{N}-1, \tau-\varepsilon),
\end{aligned}
$$

разложение которых по $\varepsilon$ имеет вид

$$
\begin{aligned}
& \hat{\overline{\mathrm{x}}}=\mathrm{x}(\mathrm{N}+1, \tau+\varepsilon) \mapsto \overline{\mathrm{x}}+\varepsilon \frac{\partial \overline{\mathrm{x}}}{\partial \tau}+\frac{\varepsilon^{2}}{2} \frac{\partial^{2} \overline{\mathrm{x}}}{\partial \tau^{2}}+\cdots, \\
& \underline{\mathrm{x}}=\mathrm{x}(\mathrm{N}-1, \tau-\varepsilon) \mapsto \underline{\mathrm{x}}-\varepsilon \frac{\partial \underline{\mathrm{x}}}{\partial \tau}+\frac{\varepsilon^{2}}{2} \frac{\partial^{2} \underline{\mathrm{x}}}{\partial \tau^{2}}-\cdots .
\end{aligned}
$$

Положения частиц $\mathrm{x}(\mathrm{N}, \tau)$ можно рассчитать с помощью собственных значений матриц (3.2) [12].

3.1. Уравнения движения и ограничения. Уравнения движения (2.33) в новых переменных $(\mathrm{N}, \tau)$ имеют вид

$$
\sum_{j=1}^{N}\left(\ln \left(\hat{\overline{\mathrm{x}}}_{j}-\mathrm{x}_{i}\right)-\ln \left(\mathrm{x}_{i}-\underline{\mathrm{x}}\right)\right)=0 .
$$

Разлагая переменную $x$ по переменной $\varepsilon$ и собирая члены перед различными степенями $\varepsilon$, получим

$$
\begin{array}{ll}
O\left(\varepsilon^{0}\right): & \sum_{j=1}^{N}\left(\ln \left(\overline{\mathrm{x}}_{j}-\mathrm{x}_{i}\right)-\ln \left(\mathrm{x}_{i}-\underline{\mathrm{x}}_{j}\right)\right)=0 \\
O\left(\varepsilon^{1}\right): & \sum_{j=1}^{N}\left[\frac{\partial \overline{\mathrm{x}}_{j}}{\partial \tau}\left(\frac{1}{\overline{\mathrm{x}}_{j}-\mathrm{x}_{i}}\right)-\frac{\partial \underline{\mathrm{x}}_{j}}{\partial \tau}\left(\frac{1}{\mathrm{x}_{i}-\underline{\mathrm{x}}_{j}}\right)\right]=0 .
\end{array}
$$

Мы оборвали ряд на $O\left(\varepsilon^{1}\right)$, однако члены высшего порядка можно получить, продолжив это разложение. Из полученного результата видно, что (3.5б) - это уравнения движения системы золотой рыбки Калоджеро с новым дискретным временем N. Уравнения (3.5в) - уравнения движения системы золотой рыбки Калоджеро с непрерывной переменной $\tau$.

Далее, осуществим предельный переход в уравнениях ограничения и соберем первые доминирующие члены:

$$
\begin{aligned}
-\frac{1}{p} & =\sum_{j=1}^{N} \frac{\partial \overline{\mathrm{x}}_{j}}{\partial \tau}\left(\frac{1}{\mathrm{x}_{i}-\overline{\mathrm{x}}_{j}}\right), \\
\frac{1}{p} & =\sum_{j=1}^{N} \frac{\partial \underline{\mathrm{x}}_{j}}{\partial \tau}\left(\frac{1}{\mathrm{x}_{i}-\underline{\mathrm{x}}_{j}}\right) .
\end{aligned}
$$

Комбинируя уравнения (3.5г) и (3.5д), непосредственно приходим к уравнениям движения (3.5в). 
3.2. Лагранжианы и соотношение замыкания. Начнем с представления лагранжиана $(2.16 \mathrm{e})$ в переменных $(\mathrm{N}, m)$ :

$$
\mathscr{L}_{(q)} \mapsto \sum_{i, j=1}^{N}\left(\mathrm{x}_{i}-\hat{\overline{\mathrm{x}}}_{j}\right) \ln \left(\mathrm{x}_{i}-\hat{\overline{\mathrm{x}}}_{j}\right)+\ln |p-\varepsilon| \sum_{i=1}^{N}\left(\mathrm{x}_{i}-\hat{\overline{\mathrm{x}}}_{i}\right) .
$$

Далее, разлагая выражение (3.6а) по переменной $\varepsilon$, получим

$$
\mathscr{L}_{(q)} \mapsto \varepsilon^{0} \mathcal{L}_{(\mathrm{N})}+\varepsilon^{1} \mathcal{L}_{(\tau)}^{(1)}+\cdots,
$$

где

$$
\begin{aligned}
& \mathcal{L}_{(\mathrm{N})}=\sum_{i, j=1}^{N}\left(\mathrm{x}_{i}-\overline{\mathrm{x}}_{j}\right) \ln \left(\mathrm{x}_{i}-\overline{\mathrm{x}}_{j}\right)+\ln |p| \sum_{i=1}^{N}\left(\mathrm{x}_{i}-\overline{\mathrm{x}}_{i}\right) \\
& \mathcal{L}_{(\tau)}^{(1)}=-\sum_{i, j=1}^{N} \frac{\partial \overline{\mathrm{x}}_{j}}{\partial \tau}\left(1+\ln \left(\mathrm{x}_{i}-\overline{\mathrm{x}}_{j}\right)\right)-\ln |p| \sum_{i=1}^{N} \frac{\partial \overline{\mathrm{x}}_{i}}{\partial \tau}-\frac{1}{p} \sum_{i=1}^{N}\left(\mathrm{x}_{i}-\overline{\mathrm{x}}_{i}\right) .
\end{aligned}
$$

Эти лагранжианы порождают уравнения движения (3.5б) и (3.5в), в чем можно убедиться подстановкой лагранжианов в следующие уравнения Эйлера-Лагранжа:

$$
\begin{aligned}
\frac{\partial \mathcal{L}_{(\mathrm{N})}}{\partial \mathrm{x}}+\frac{\partial \mathcal{L}_{(\mathrm{N})}}{\partial \overline{\mathrm{x}}} & =0 \\
\frac{\partial \mathcal{L}_{(\tau)}^{(1)}}{\partial \mathrm{x}}+\frac{\partial \mathcal{L}_{(\tau)}^{(1)}}{\partial \overline{\mathrm{x}}}-\frac{d}{d \tau}\left(\frac{\partial \mathcal{L}_{(\tau)}^{(1)}}{\partial(\partial \overline{\mathrm{x}} / \partial \tau)}\right) & =0
\end{aligned}
$$

Кроме того, ограничения (3.5г) и (3.5д) вытекают из уравнений Эйлера-Лагранжа по переменной $\bar{x}$ для лагранжиана $\mathcal{L}_{(\tau)}^{(1)}$

$$
\frac{\partial \mathcal{L}_{(\tau)}^{(1)}}{\partial \overline{\mathrm{x}}}-\frac{d}{d \tau}\left(\frac{\partial \mathcal{L}_{(\tau)}^{(1)}}{\partial(\partial \overline{\mathrm{x}} / \partial \tau)}\right)=0
$$

Далее, перейдем к непрерывному пределу в соотношении замыкания и возьмем первые два доминирующих члена по степеням $\varepsilon$ :

$$
O\left(\varepsilon^{1}\right): \quad \frac{\partial \mathcal{L}_{(\mathrm{N})}}{\partial \tau}=\mathcal{L}_{(\tau)}^{(1)}-\underline{\mathcal{L}_{(\tau)}^{(1)}} .
$$

Уравнение (3.63) представляет собой соотношение замыкания между дискретным лагранжианом $\mathcal{L}_{(\mathrm{N})}$ и непрерывным лагранжианом $\mathcal{L}_{(\tau)}^{(1)}$. Это соотношение гарантирует инвариантность действия в пространстве независимых переменных, смешивающем дискретную переменную $\mathrm{N}$ и непрерывную переменную $\tau$.

\section{4. ПОЛНЫЙ НЕПРЕРЫВНЫЙ ПРЕДЕЛ}

Завершим процесс перехода к непрерывному пределу. Начнем с разложения (3.2) по переменной $p$

$$
\begin{aligned}
\mathbf{Y}(\mathrm{N}, \tau) \mapsto & \mathbf{Y}(0,0)+\mathrm{N} \frac{\boldsymbol{\Lambda}}{p}\left(1-\frac{\boldsymbol{\Lambda}}{p}+\left(\frac{\boldsymbol{\Lambda}}{p}\right)^{2}-\left(\frac{\boldsymbol{\Lambda}}{p}\right)^{3}+\cdots\right)+ \\
& +\tau \boldsymbol{\Lambda}\left(\frac{1}{p^{2}}-\frac{2 \boldsymbol{\Lambda}}{p^{3}}+\frac{3 \boldsymbol{\Lambda}^{2}}{p^{4}}-\cdots\right)
\end{aligned}
$$


а затем соберем члены перед степенями $\boldsymbol{\Lambda}$ :

$$
\mathbf{Y}(\mathrm{N}, \tau) \mapsto \mathbf{Y}\left(t_{1}, t_{2}, t_{3}, \ldots, t_{N}\right)=\mathbf{Y}(0,0)+\boldsymbol{\Lambda} t_{1}+\boldsymbol{\Lambda}^{2} t_{2}+\boldsymbol{\Lambda}^{3} t_{3}+\cdots+\boldsymbol{\Lambda}^{N} t_{N},
$$

где

$$
t_{1}=\frac{\tau}{p^{2}}+\frac{\mathrm{N}}{p}, \quad t_{2}=-\frac{2 \tau}{p^{3}}-\frac{\mathrm{N}}{p^{2}}, \quad \ldots, \quad t_{N}=(-1)^{N+1}\left(\frac{N \tau}{p^{N+1}}+\frac{\mathrm{N}}{p^{N}}\right) .
$$

Положение $i$-й частицы $X_{i}\left(t_{1}, t_{2}, \ldots, t_{N}\right)$ можно определить с помощью собственных значений матрицы (4.2).

Используя эти новые непрерывные переменные, найдем

$$
\begin{aligned}
\frac{\partial \mathrm{x}_{i}}{\partial \tau} & =\frac{\partial X_{i}}{\partial t_{1}} \frac{\partial t_{1}}{\partial \tau}+\frac{\partial X_{i}}{\partial t_{2}} \frac{\partial t_{2}}{\partial \tau}+\frac{\partial X_{i}}{\partial t_{3}} \frac{\partial t_{3}}{\partial \tau}+\cdots+\frac{\partial X_{i}}{\partial t_{N}} \frac{\partial t_{N}}{\partial \tau}= \\
& =\frac{1}{p^{2}} \frac{\partial X_{i}}{\partial t_{1}}-\frac{2}{p^{3}} \frac{\partial X_{i}}{\partial t_{2}}+\frac{3}{p^{4}} \frac{\partial X_{i}}{\partial t_{3}}+\cdots+\frac{(-1)^{N+1} N}{p^{N+1}} \frac{\partial X_{i}}{\partial t_{N}}
\end{aligned}
$$

и

$$
\begin{aligned}
\mathrm{x}_{i}(\mathrm{~N} \pm 1)= & \exp \left[ \pm \frac{\partial}{p \partial t_{1}} \mp \frac{\partial}{p^{2} \partial t_{2}} \pm \frac{\partial}{p^{3} \partial t_{3}} \mp \frac{\partial}{p^{4} \partial t_{4}} \pm \cdots\right] X_{i}= \\
= & X_{i} \pm \frac{1}{p} \frac{\partial X_{i}}{\partial t_{1}}+\frac{1}{p^{2}}\left(\frac{1}{2} \frac{\partial^{2} X_{i}}{\partial t_{1}^{2}} \mp \frac{\partial X_{i}}{\partial t_{2}}\right)+\frac{1}{p^{3}}\left( \pm \frac{1}{6} \frac{\partial^{3} X_{i}}{\partial t_{1}^{3}}-\frac{\partial^{2} X_{i}}{\partial t_{1} \partial t_{2}} \pm \frac{\partial X_{i}}{\partial t_{3}}\right)+ \\
& +\frac{1}{p^{4}}\left(\frac{1}{24} \frac{\partial^{4} X_{i}}{\partial t_{1}^{4}} \mp \frac{1}{2} \frac{\partial^{3} X_{i}}{\partial t_{1}^{2} \partial t_{2}}+\frac{1}{2} \frac{\partial^{2} X_{i}}{\partial t_{2}^{2}}+\frac{\partial^{2} X_{i}}{\partial t_{1} \partial t_{3}} \mp \frac{\partial X_{i}}{\partial t_{4}}\right)+O\left(\frac{1}{p^{5}}\right) .
\end{aligned}
$$

Для простоты далее в настоящем разделе ограничимся случаями первых двух времен.

4.1. Уравнения движения. Разлагая уравнение (3.5б), получим

$$
\begin{gathered}
O\left(\frac{1}{p}\right): \quad \frac{\partial^{2} X_{i}}{\partial t_{1}^{2}}-2 \sum_{\substack{j=1 \\
j \neq i}}^{N} \frac{\partial X_{i}}{\partial t_{1}} \frac{\partial X_{j}}{\partial t_{1}} \frac{1}{X_{i}-X_{j}}=0, \\
O\left(\frac{1}{p^{2}}\right): \quad \frac{\partial^{2} X_{i}}{\partial t_{1} \partial t_{2}}-2 \sum_{\substack{j=1 \\
j \neq i}}^{N} \frac{\partial X_{i}}{\partial t_{1}} \frac{\partial X_{j}}{\partial t_{2}} \frac{1}{X_{i}-X_{j}}=0 .
\end{gathered}
$$

Уравнения (4.6) - это обычные уравнения движения системы золотой рыбки Калоджеро. Уравнения (4.7) можно считать уравнениями движения следующей системы рассматриваемой иерархии. Остальные уравнения движения иерархии можно получить, продолжив разложение.

4.2. Лагранжианы. Уравнениям движения (4.6) и (4.7) соответствуют следующие лагранжианы:

$$
\begin{aligned}
& L_{\left(t_{1}\right)}=\sum_{i=1}^{N}\left(\frac{\partial X_{i}}{\partial t_{1}} \ln \left|\frac{\partial X_{i}}{\partial t_{1}}\right|+\frac{\partial X_{i}}{\partial t_{1}}\right)+\sum_{i \neq j}^{N} \frac{\partial X_{j}}{\partial t_{1}} \ln \left|X_{i}-X_{j}\right|, \\
& L_{\left(t_{2}\right)}=\sum_{i=1}^{N}\left(\frac{\partial X_{i}}{\partial t_{2}} \ln \left|\frac{\partial X_{i}}{\partial t_{1}}\right|+2 \frac{\partial X_{i}}{\partial t_{2}}\right)+\sum_{i \neq j}^{N} \frac{\partial X_{j}}{\partial t_{2}} \ln \left|X_{i}-X_{j}\right|,
\end{aligned}
$$




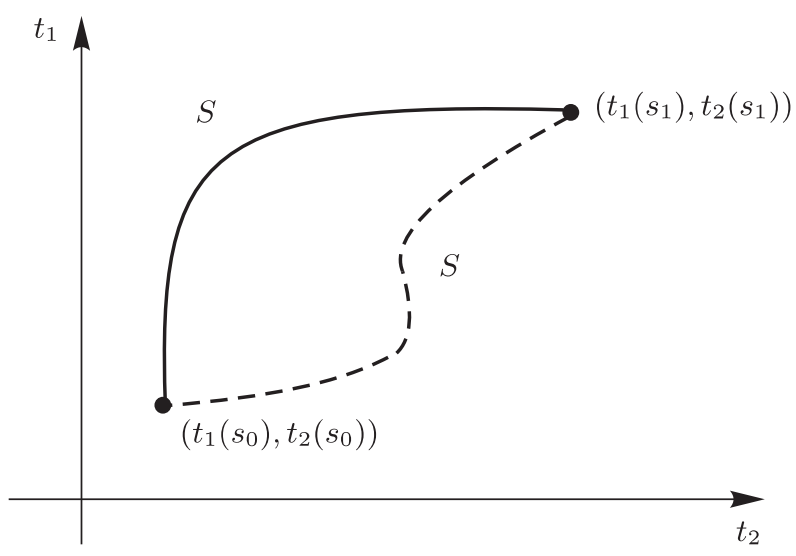

Рис. 3. Деформация непрерывной кривой в пространстве независимых переменных $\left(t_{1}(s), t_{2}(s)\right)$, где $s$ - переменная, параметризующая время: $s_{0}<s<$ $s_{1}$. Инвариантность действия возникает вследствие того, что $\delta S=S^{\prime}-S=0$, а это приводит к соотношению замыкания.

при этом уравнения Эйлера-Лагранжа имеют вид

$$
\begin{aligned}
& \frac{\partial L_{\left(t_{1}\right)}}{\partial X_{i}}-\frac{\partial}{\partial t_{1}}\left(\frac{\partial L_{\left(t_{1}\right)}}{\partial\left(\partial X_{i} / \partial t_{1}\right)}\right)=0, \\
& \frac{\partial L_{\left(t_{2}\right)}}{\partial X_{i}}-\frac{\partial}{\partial t_{2}}\left(\frac{\partial L_{\left(t_{2}\right)}}{\partial\left(\partial X_{i} / \partial t_{2}\right)}\right)=0 .
\end{aligned}
$$

Лагранжианы (4.8) и (4.9) можно получить и другим способом, а именно в результате полного непрерывного предельного перехода в действии, как в случае систем Калоджеро-Мозера и Рейсенарса-Шнайдера (см. [4], [7]).

Кроме того, найдем следующее соотношение замыкания для этих двух лагранжианов:

$$
\frac{\partial L_{\left(t_{2}\right)}}{\partial t_{1}}=\frac{\partial L_{\left(t_{1}\right)}}{\partial t_{2}} .
$$

И снова это соотношение гарантирует инвариантность действия

$$
S=\int_{\Gamma}\left(L_{\left(t_{1}\right)} d t_{1}+L_{\left(t_{2}\right)} d t_{2}\right)
$$

относительно локальных деформаций кривой $Г$ в пространстве независимых переменных $\left(t_{1}, t_{2}\right)$ (см. рис. 3$)$.

ЗАмЕчАние 2. Мы видим, что лагранжианы $L_{\left(t_{1}\right)}$ и $L_{\left(t_{2}\right)}$ имеют одни и те же переменные-импульсы

$$
\pi_{i}=\ln \left|\frac{\partial X_{i}}{\partial t_{1}}\right|+1+\sum_{\substack{j=1 \\ j \neq i}}^{N} \ln \left(X_{i}-X_{j}\right)
$$


к такому заключению можно прийти и в результате перехода к непрерывному пределу в уравнениях (2.18):

$$
\begin{gathered}
\pi_{i}=\sum_{j=1}^{N} \ln \left(x_{j}-\hat{x}_{i}\right)+1+\ln |q|, \\
\text { асимметричный предел } \pi_{i}=\sum_{j=1}^{N} \ln \left(\mathrm{x}_{j}-\bar{x}_{i}\right)+1+\ln |p|, \\
\text { полный предел } \pi_{i}=\ln \left|\frac{\partial X_{i}}{\partial t_{1}}\right|+1+\sum_{\substack{j=1, j \neq i}}^{N} \ln \left(X_{i}-X_{j}\right) .
\end{gathered}
$$

Здесь рассматриваются только доминирующие члены.

Используя переменные (4.14), получим

$$
h_{i}^{2}=\frac{\partial X_{i}}{\partial t_{1}}=\frac{e^{\pi_{i}-1}}{\prod_{j \neq i}\left(X_{i}-X_{j}\right)},
$$

а матрица $\mathbf{L}_{0}$ преобразуется к виду

$$
\mathbf{L}_{0}=\sum_{i, j=1}^{N} h_{i} h_{j} E_{i j}=\sum_{i, j=1}^{N} \sqrt{\frac{\partial X_{i}}{\partial t_{1}} \frac{\partial X_{j}}{\partial t_{1}}} E_{i j}
$$

при этом

$$
\operatorname{Tr} \mathbf{L}_{0}=H_{\left(t_{1}\right)}=\sum_{i=1}^{N} \frac{\partial X_{i}}{\partial t_{1}}=\sum_{i=1}^{N} \frac{e^{\pi_{i}-1}}{\prod_{j \neq i}\left(X_{i}-X_{j}\right)},
$$

что является первым гамильтонианом иерархии. Связь с лагранжианом $L_{\left(t_{1}\right)}$ можно установить с помощью преобразования Лежандра

$$
\begin{aligned}
L_{\left(t_{1}\right)} & =\sum_{i=1}^{N} \pi_{i} \frac{\partial X_{i}}{\partial t_{1}}-H_{\left(t_{1}\right)}=\sum_{i=1}^{N}\left(\pi_{i}-1\right) \frac{\partial X_{i}}{\partial t_{1}}= \\
& =\sum_{i=1}^{N} \frac{\partial X_{i}}{\partial t_{1}} \ln \left|\frac{\partial X_{i}}{\partial t_{1}}\right|+\sum_{i \neq j}^{N} \frac{\partial X_{j}}{\partial t_{1}} \ln \left|X_{i}-X_{j}\right|
\end{aligned}
$$

и используя переменные (4.14).

$\mathrm{K}$ сожалению, матрицу Лакса $\mathbf{L}_{0}$ следует считать ложной матрицей Лакса, поскольку она порождает только первую сохраняющуюся при движении величину [10]. Для получения остальных гамильтонианов нужен другой метод [11]. Рассмотрим второй гамильтониан в виде

$$
H_{\left(t_{2}\right)}=\sum_{i=1}^{N} \frac{e^{\pi_{i}-1}}{\prod_{j \neq i}\left(X_{i}-X_{j}\right)} \sum_{\substack{j=1, j \neq i}}^{N} X_{j}
$$

и

$$
\frac{\partial X_{i}}{\partial t_{2}}=\frac{\partial H_{\left(t_{2}\right)}}{\partial \pi_{i}}=\frac{e^{\pi_{i}-1}}{\prod_{j \neq i}\left(X_{i}-X_{j}\right)} \sum_{\substack{j=1, j \neq i}}^{N} X_{j} .
$$


Выполнив преобразование Лежандра, получим

$$
\begin{aligned}
L_{\left(t_{2}\right)} & =\sum_{i=1}^{N} \pi_{i} \frac{\partial X_{i}}{\partial t_{2}}-H_{\left(t_{2}\right)}=\sum_{i=1}^{N}\left(\pi_{i}-1\right) \frac{\partial X_{i}}{\partial t_{2}}= \\
& =\sum_{i=1}^{N} \frac{\partial X_{i}}{\partial t_{2}} \ln \left|\frac{\partial X_{i}}{\partial t_{1}}\right|+\sum_{i \neq j} \frac{\partial X_{j}}{\partial t_{1}} \ln \left|X_{i}-X_{j}\right|
\end{aligned}
$$

что является вторым лагранжианом (с точностью до члена с полной производной).

\section{5. СВЯЗЬ С РЕШЕТОЧНОЙ СИСТЕМОЙ КАДОМЦЕВА-ПЕТВИАШВИЛИ}

В работе [12] система Калоджеро-Мозера с дискретным временем была естественным образом получена при исследовании полюсного решения полудискретного уравнения Кадомцева-Петвиашвили. В противоположность этому, система Руджинарса-Шнайдера с дискретным временем была получена из анзаца пары Лакса [13]. С другой стороны, в работе [7] была установлена связь между системой Руджинарса-Шнайдера и решеточной системой Кадомцева-Петвиашвили. По аналогии с системой Руджинарса-Шнайдера начнем вывод системы золотой рыбки Калоджеро с дискретным временем из анзаца пары Лакса. В этом разделе исследуется связь между решеточной системой Кадомцева-Петвиашвили и системой золотой рыбки Калоджеро.

Рассмотрим тау-функцию в виде характеристического полинома:

$$
\tau(\xi)=\operatorname{det}(\xi I-\mathbf{Y})
$$

где функция $\mathbf{Y}=\mathbf{Y}(n, m, h)$ дана выражением (2.14) и является функцией трех дискретных переменных. При этом возникают соотношения

$$
\begin{aligned}
& \tilde{\mathbf{Y}}-\mathbf{Y}=\tilde{\mathbf{r}} \mathbf{s}^{\mathrm{T}}, \\
& \widehat{\mathbf{Y}}-\mathbf{Y}=\hat{\mathbf{r}} \mathbf{s}^{\mathrm{T}}, \\
& \overline{\mathbf{Y}}-\mathbf{Y}=\overline{\mathbf{r}} \mathbf{s}^{\mathrm{T}},
\end{aligned}
$$

где $\mathbf{r}$ и $\mathbf{s}-$ функции дискретных переменных, введенные с помощью следующих сдвиговых соотношений (см. (2.10)):

$$
\begin{array}{ll}
(p I+\boldsymbol{\Lambda}) \cdot \tilde{\mathbf{r}}=\mathbf{r}, & \mathbf{s}^{\mathrm{T}} \cdot(p I+\boldsymbol{\Lambda})=\tilde{\mathbf{s}}^{\mathrm{T}}, \\
(q I+\boldsymbol{\Lambda}) \cdot \hat{\mathbf{r}}=\mathbf{r}, & \mathbf{s}^{\mathrm{T}} \cdot(q I+\boldsymbol{\Lambda})=\hat{\mathbf{s}}^{\mathrm{T}}, \\
(r I+\boldsymbol{\Lambda}) \cdot \overline{\mathbf{r}}=\mathbf{r}, & \mathbf{s}^{\mathrm{T}} \cdot(r I+\boldsymbol{\Lambda})=\overline{\mathbf{s}}^{\mathrm{T}} .
\end{array}
$$

Чтобы вывести решеточное уравнение Кадомцева-Петвиашвили, проведем сначала следующее вычисление:

$$
\begin{aligned}
\tilde{\tau}(\xi) & =\operatorname{det}\left(\xi-\mathbf{Y}-\tilde{\mathbf{r}} \mathbf{s}^{\mathrm{T}}\right)= \\
& =\operatorname{det}\left((\xi-\mathbf{Y})\left(1-\tilde{\mathbf{r}} \mathbf{s}^{\mathrm{T}}(\xi-\mathbf{Y})^{-1}\right)\right)= \\
& =\tau(\xi)\left(1-\mathbf{s}^{\mathrm{T}}(\xi-\mathbf{Y})^{-1} \tilde{\mathbf{r}}\right),
\end{aligned}
$$

в результате получим

$$
\frac{\tilde{\tau}(\xi)}{\tau(\xi)}=\mathbf{v}_{p}(\xi)
$$


где функция $\mathbf{v}_{p}$ задана выражением

$$
\mathbf{v}_{a}(\xi):=1-\mathbf{s}^{\mathrm{T}}(\xi-\mathbf{Y})^{-1}(a+\boldsymbol{\Lambda})^{-1} \mathbf{r}
$$

с произвольным параметром $a$.

Взаимосвязь, обратную соотношению (5.4), получим с помощью аналогичного расчета:

$$
\begin{aligned}
\tau(\xi) & =\operatorname{det}\left(\xi-\tilde{\mathbf{Y}}+\tilde{\mathbf{r}} \mathbf{s}^{\mathrm{T}}\right)= \\
& =\operatorname{det}\left((\xi-\tilde{\mathbf{Y}})\left(1+\tilde{\mathbf{r}} \mathbf{s}^{\mathrm{T}}(\xi-\tilde{\mathbf{Y}})^{-1}\right)\right)= \\
& =\tilde{\tau}(\xi)\left(1+\mathbf{s}^{\mathrm{T}}(\xi-\tilde{\mathbf{Y}})^{-1} \tilde{\mathbf{r}}\right)
\end{aligned}
$$

который приводит к уравнению

$$
\frac{\tau(\xi)}{\tilde{\tau}(\xi)}=\widetilde{\mathbf{w}}_{p}(\xi),
$$

где функция $\mathbf{w}_{p}$ задана выражением

$$
\mathbf{w}_{a}(\xi):=1+\mathbf{s}^{\mathrm{T}}(a+\boldsymbol{\Lambda})^{-1}(\xi-\mathbf{Y})^{-1} \mathbf{r}
$$

с произвольным параметром $a$.

Из уравнений (5.4) и (5.6) получим соотношение

$$
\frac{\tau(\xi)}{\tilde{\tau}(\xi)}=\widetilde{\mathbf{w}}_{p}(\xi)=\frac{1}{\mathbf{v}_{p}(\xi)} .
$$

С помощью расчетов подобного рода можно получить соотношения того же типа и для других дискретных направлений:

$$
\begin{aligned}
\frac{\tau(\xi)}{\hat{\tau}(\xi)} & =\widehat{\mathbf{w}}_{q}(\xi)=\frac{1}{\mathbf{v}_{q}(\xi)}, \\
\frac{\tau(\xi)}{\bar{\tau}(\xi)} & =\overline{\mathbf{w}}_{r}(\xi)=\frac{1}{\mathbf{v}_{r}(\xi)} .
\end{aligned}
$$

Введем теперь $N$-компонентные векторы

$$
\begin{aligned}
\mathbf{u}_{a}(\xi) & =(\xi-\mathbf{Y})^{-1}(a+\boldsymbol{\Lambda})^{-1} \mathbf{r}, \\
{ }^{\mathbf{t}} \mathbf{u}_{b}(\xi) & =\mathbf{s}^{\mathrm{T}}(b+\boldsymbol{\Lambda})^{-1}(\xi-\mathbf{Y})^{-1},
\end{aligned}
$$

а также скалярные переменные

$$
S_{a b}(\xi)=\mathbf{s}^{\mathrm{T}}(b+\boldsymbol{\Lambda})^{-1}(\xi-\mathbf{Y})^{-1}(a+\boldsymbol{\Lambda})^{-1} \mathbf{r} .
$$

Уравнение (5.10а) можно представить в виде

$$
\mathbf{u}_{a}(\xi)=(p-a) \tilde{\mathbf{u}}_{a}(\xi)+\mathbf{v}_{a}(\xi) \tilde{\mathbf{u}}_{0}(\xi),
$$

где $\mathbf{u}_{0}(\xi)=(\xi-\mathbf{Y})^{-1} \mathbf{r}$, при этом уравнение (5.10б) можно также представить в виде

$$
\widetilde{\mathbf{t}_{\mathbf{u}}}(\xi)=(p-b) \widetilde{\widetilde{\mathbf{t}}^{\mathbf{u}}}(\xi)+\widetilde{\mathbf{w}}_{b}(\xi) \widetilde{\mathbf{t} \mathbf{u}_{0}}(\xi),
$$

где ${ }^{\mathbf{t}} \mathbf{u}_{0}(\xi)=\mathbf{s}^{\mathrm{T}}(\xi-\mathbf{Y})^{-1}$. 
Другой тип связей можно получить путем умножения уравнения (5.11) слева на $\tilde{\mathbf{s}}^{\mathrm{T}}(b+\boldsymbol{\Lambda})^{-1}$. Имеем

$$
\begin{aligned}
& \tilde{\mathbf{s}}^{\mathrm{T}}(b+\boldsymbol{\Lambda})^{-1} \mathbf{u}_{a}(\xi)=(p-a) \tilde{\mathbf{s}}^{\mathrm{T}}(b+\boldsymbol{\Lambda})^{-1} \tilde{\mathbf{u}}_{a}(\xi)++\mathbf{v}_{a}(\xi) \tilde{\mathbf{s}}^{\mathrm{T}}(b+\boldsymbol{\Lambda})^{-1} \tilde{\mathbf{u}}_{0}(\xi), \\
& \mathbf{s}^{\mathrm{T}}(p+\boldsymbol{\Lambda})(b+\boldsymbol{\Lambda})^{-1} \mathbf{u}_{a}(\xi)=(p-a) \widetilde{S}_{a b}(\xi)+\mathbf{v}_{a}(\xi) \widetilde{\mathbf{w}}_{b}(\xi), \\
& \mathbf{v}_{a}(\xi) \widetilde{\mathbf{w}}_{b}(\xi)=1+(p-b) S_{a b}(\xi)-(p-a) \widetilde{S}_{a b}(\xi) .
\end{aligned}
$$

Запишем остальные уравнения в направлениях других дискретных времен:

$$
\begin{aligned}
\mathbf{v}_{a}(\xi) \widehat{\mathbf{w}}_{b}(\xi) & =1+(q-b) S_{a b}(\xi)-(q-a) \widehat{S}_{a b}(\xi), \\
\mathbf{v}_{a}(\xi) \overline{\mathbf{w}}_{b}(\xi) & =1+(r-b) S_{a b}(\xi)-(r-a) \bar{S}_{a b}(\xi) .
\end{aligned}
$$

Воспользовавшись тождеством

$$
\frac{\widetilde{\widetilde{\mathbf{w}}}_{b}(\xi) \overline{\mathbf{v}}_{a}(\xi)}{\widehat{\widehat{\mathbf{w}}}_{b}(\xi) \overline{\mathbf{v}}_{a}(\xi)}=\frac{\widetilde{\mathbf{w}}_{b}(\xi) \tilde{\mathbf{v}}_{a}(\xi)}{\widehat{\widehat{\mathbf{w}}}_{b}(\xi) \hat{\mathbf{v}}_{a}(\xi)} \frac{\widehat{\widetilde{\mathbf{w}}}_{b}(\xi) \hat{\mathbf{v}}_{a}(\xi)}{\widehat{\widetilde{\mathbf{w}}}_{b}(\xi) \tilde{\mathbf{v}}_{a}(\xi)},
$$

можно вывести соотношение

$$
\begin{aligned}
& \frac{1+(p-b) \bar{S}_{a b}(\xi)-(p-a) \widetilde{\widetilde{S}}_{a b}(\xi)}{1+(q-b) \bar{S}_{a b}(\xi)-(q-a) \widehat{\bar{S}}_{a b}(\xi)}= \\
& \quad=\frac{1+(r-b) \widetilde{S}_{a b}(\xi)-(r-a) \widetilde{\bar{S}}_{a b}(\xi)}{1+(q-b) \widetilde{S}_{a b}(\xi)-(q-a) \widehat{\widetilde{S}}_{a b}(\xi)} \frac{1+(p-b) \widehat{S}_{a b}(\xi)-(p-a) \widehat{\widetilde{S}}_{a b}(\xi)}{1+(r-b) \widehat{S}_{a b}(\xi)-(r-a) \widehat{\bar{S}}_{a b}(\xi)},
\end{aligned}
$$

которое является трехмерным решеточным уравнением, впервые полученным в работе [14] и называемым решеточным уравнением Швариа-Кадомиева-Петвиашвили [15].

Умножив теперь уравнение (5.11) слева на $\widetilde{\mathbf{s}}^{\mathrm{T}}$, получим

$$
\begin{aligned}
\tilde{\mathbf{s}}^{\mathrm{T}} \mathbf{u}_{a}(\xi) & =(p-a) \tilde{\mathbf{s}}^{\mathrm{T}} \tilde{\mathbf{u}}_{a}(\xi)+\mathbf{v}_{a}(\xi) \tilde{\mathbf{s}}^{\mathrm{T}} \tilde{\mathbf{u}}_{0}(\xi), \\
\mathbf{s}^{\mathrm{T}}(p+\boldsymbol{\Lambda}) \mathbf{u}_{a}(\xi) & =(p-a)\left(1-\widetilde{\mathbf{v}}_{a}(\xi)\right)+\mathbf{v}_{a}(\xi) \tilde{\mathbf{s}}^{\mathrm{T}} \tilde{\mathbf{u}}_{0}(\xi) .
\end{aligned}
$$

Введем

$$
u(\xi)=\mathbf{s}^{\mathrm{T}}(\xi-\mathbf{Y})^{-1} \mathbf{r}
$$

и перепишем уравнение (5.17) в виде

$$
(p+\tilde{u}(\xi)) \mathbf{v}_{a}(\xi)-(p-a) \tilde{\mathbf{v}}_{a}(\xi)=a+\mathbf{s}^{\mathrm{T}} \boldsymbol{\Lambda} \mathbf{u}_{a}(\xi)
$$

Другие два соотношения, связанные с другими дискретными направлениями, получим автоматически:

$$
\begin{aligned}
& (q+\hat{u}(\xi)) \mathbf{v}_{a}(\xi)-(q-a) \hat{\mathbf{v}}_{a}(\xi)=a+\mathbf{s}^{\mathrm{T}} \boldsymbol{\Lambda} \mathbf{u}_{a}(\xi) \\
& (r+\bar{u}(\xi)) \mathbf{v}_{a}(\xi)-(r-a) \overline{\mathbf{v}}_{a}(\xi)=a+\mathbf{s}^{\mathrm{T}} \boldsymbol{\Lambda} \mathbf{u}_{a}(\xi)
\end{aligned}
$$

Исключая член $\mathbf{s}^{\mathrm{T}} \boldsymbol{\Lambda} \mathbf{u}_{a}(\xi)$, выведем соотношения

$$
\begin{aligned}
& (p-q+\tilde{u}(\xi)-\hat{u}(\xi)) \mathbf{v}_{a}(\xi)=(p-a) \tilde{\mathbf{v}}_{a}(\xi)-(q-a) \hat{\mathbf{v}}_{a}(\xi), \\
& (p-r+\tilde{u}(\xi)-\bar{u}(\xi)) \mathbf{v}_{a}(\xi)=(p-a) \tilde{\mathbf{v}}_{a}(\xi)-(r-a) \overline{\mathbf{v}}_{a}(\xi), \\
& (r-q+\bar{u}(\xi)-\hat{u}(\xi)) \mathbf{v}_{a}(\xi)=(r-a) \overline{\mathbf{v}}_{a}(\xi)-(q-a) \hat{\mathbf{v}}_{a}(\xi) .
\end{aligned}
$$


Положим теперь $p=a$, тогда уравнения (5.21a) и (5.21б) принимают вид

$$
\begin{aligned}
& p-q+\tilde{u}(\xi)-\hat{u}(\xi)=-(q-p) \frac{\hat{\mathbf{v}}_{p}(\xi)}{\mathbf{v}_{p}(\xi)}, \\
& p-r+\tilde{u}(\xi)-\bar{u}(\xi)=-(r-p) \frac{\overline{\mathbf{v}}_{p}(\xi)}{\mathbf{v}_{p}(\xi)} .
\end{aligned}
$$

Комбинируя уравнения (5.22а) и (5.22б), получим уравнение

$$
\frac{p-q+\tilde{u}(\xi)-\hat{u}(\xi)}{p-r+\tilde{u}(\xi)-\bar{u}(\xi)}=\frac{p-q+\tilde{\bar{u}}(\xi)-\hat{\bar{u}}(\xi)}{p-r+\hat{\tilde{u}}(\xi)-\hat{\bar{u}}(\xi)}
$$

которое представляет собой решеточное уравнение Кадомиева-Петвиашвили [14] (см. также [16]).

Используя определение функции $\mathbf{v}_{p}(\xi)$ в виде (5.4), перепишем уравнения (5.22a) и (5.22б) через тау-функцию:

$$
\begin{aligned}
& p-q+\tilde{u}(\xi)-\hat{u}(\xi)=-(q-p) \frac{\hat{\tilde{\tau}}(\xi)}{\hat{\tau}(\xi)} \frac{\tau(\xi)}{\tilde{\tau}(\xi)}, \\
& p-r+\tilde{u}(\xi)-\bar{u}(\xi)=-(r-p) \frac{\tilde{\tilde{\tau}}(\xi)}{\bar{\tau}(\xi)} \frac{\tau(\xi)}{\tilde{\tau}(\xi)} .
\end{aligned}
$$

Если положить $r=a$, то из уравнения (5.21в) получим также

$$
r-q+\bar{u}(\xi)-\hat{u}(\xi)=-(q-r) \frac{\hat{\bar{\tau}}(\xi)}{\hat{\tau}(\xi)} \frac{\tau(\xi)}{\bar{\tau}(\xi)} .
$$

Комбинируя уравнения (5.24a), (5.24б) и (5.25), получим уравнение

$$
(p-q) \hat{\tilde{\tau}}(\xi) \bar{\tau}(\xi)+(r-p) \tilde{\bar{\tau}}(\xi) \hat{\tau}(\xi)+(r-q) \hat{\bar{\tau}}(\xi) \tilde{\tau}(\xi)=0
$$

которое является билинейным решеточным уравнением Кадомцева-Петвиашвили (первоначально названным дискретным аналогом обобщенного уравнения Тоды, см. [17]).

Нам удалось установить связь системы золотой рыбки Калоджеро с решеточной системой Кадомцева-Петвиашвили. Это завершает описание связи дискретных одномерных интегрируемых систем многих тел, таких как система Калоджеро-Мозера и система Руджинарса-Шнайдера, с решеточными системами Кадомцева-Петвиашвили.

\section{6. ВЫВОДЫ}

Подробно изучен еще один пример лагранжиана в виде 1-формы с помощью рациональной системы золотой рыбки Калоджеро. В этом примере на уровне дискретного времени система была получена из анзаца пары Лакса (а не с помощью процесса полюсной редукции системы Кадомцева-Петвиашвили, приводящей к системе Калоджеро-Мозера с дискретным временем) подобно системе Руджинарса-Шнайдера с дискретным временем. В результате получены системы золотой рыбки Калоджеро с дискретным временем, связанные с разными дискретными переменными. Совместность этих двух дискретных направлений приводит к ограничениям, объясняющим, каким образом система переводится от одной дискретной переменной 
к другой. Вариация дискретного действия по отношению к дискретному времени приводит к соотношению замыкания, которое гарантирует неизменность значения действия при локальных деформациях дискретной кривой в пространстве дискретных времен. После этого был осуществлен переход к непрерывному пределу, а именно к несимметричному пределу и к полному непрерывному пределу, с целью порождения иерархии лагранжианов для рассматриваемой системы. Любопытно, что эти лагранжианы являются функциями многих времен (число времен в этом случае может достигать числа частиц). Непрерывное соотношение замыкания системы, которое получается непосредственно из вариационного принципа по отношению к временны́ переменным, и в этом случае гарантирует инвариантность действия при локальных деформациях непрерывной кривой в пространстве непрерывных переменных. Более того, с помощью структуры точного решения системы золотой рыбки Калоджеро была установлена связь между системой золотой рыбки Калоджеро и решеточной системой Кадомцева-Петвиашвили.

Благодарности. С. Ю-Конг искренне признателен Thailand Research Fund (TRF) за поддержку (грант № TRG5680081).

\section{Список литературы}

[1] S. B. Lobb, F. W. Nijhoff, J. Phys. A: Math. Theor., 42:45 (2009), 454013, 18 pp., arXiv: 0903.4086.

[2] S. B. Lobb, F. W. Nijhoff, J. Phys. A: Math. Theor., 43:7 (2010), 072003, 11 pp.

[3] S. B. Lobb, F. W. Nijhoff, G. R. W. Quispel, J. Phys. A: Math. Theor., 42:47 (2009), 472002, $11 \mathrm{pp}$.

[4] F. Calogero, J. Math. Phys., 10:12 (1969), 2191-2196.

[5] F. Calogero, J. Math. Phys., 12:3 (1971), 419-436.

[6] S. Yoo-Kong, S. B. Lobb, F. W. Nijhoff, J. Phys. A: Math. Theor., 44:36 (2011), 365203, $39 \mathrm{pp}$.

[7] S. Yoo-Kong, F. W. Nijhoff, Discrete-time Ruijsenaars-Schneider system and Lagrangian 1-form structure, arXiv: 1112.4576.

[8] Yu. B. Suris, J. Geom. Mech., 5:3 (2013), 365-379.

[9] R. Boll, M. Petrera, Yu. B. Suris, J. Phys. A: Math. Theor., 46:27 (2013), 275204, 26 pp., arXiv: 1408.2405.

[10] F. Calogero, Phys. D, 152-153 (2001), 78-84.

[11] Yu. B. Suris, J. Nonlinear Math. Phys., 12:supp. 1 (2005), 633-647.

[12] F. W. Nijhoff, P. Gen-Di, Phys. Lett. A, 191:1-2 (1994), 101-107.

[13] F. W. Nijhoff, O. Ragnisco, V. Kuznetsov, Commun. Math. Phys., 176:3 (1996), 681-700.

[14] F. W. Nijhoff, H. W. Capel, G. L. Wiersma, G. R.W. Quispel, Phys. Lett. A, 105:6 (1984), $267-272$.

[15] I. Ya. Dorfman, F. W. Nijhoff, Phys. Lett. A, 157:2-3 (1991), 107-112.

[16] F. W. Nijhoff, H. W. Capel, G. L. Wiersma, "Integrable lattice systems in two and three dimensions", Geometric Aspects of the Einstein Equations and Integrable Systems (Scheveningen, The Netherlands, August 26-31, 1984), Lecture Notes in Physics, 239, ed. R. Martini, Springer, Berlin-New York, 1985, 263-302.

[17] R. Hirota, J. Phys. Soc. Japan, 50:11 (1981), 3785-3791. 